\title{
Comparison of QT/RR Relation Based on a 15-s Averaged ECG and a Single Beat ECG During Atrial Fibrillation
}

\author{
Akira Fujiki, MD, PhD; Masao Sakabe, MD, PhD
}

\begin{abstract}
Background: The aim of this study was to compare QT/RR relation based on a 15-s averaged beat ECG with a single beat ECG during atrial fibrillation (AF) and to determine which was better to estimate the QT interval after sinus restoration.
\end{abstract}

\begin{abstract}
Methods and Results: QT and RR intervals were measured using an automatic QT analyzing system in 33 patients who had both AF and sinus rhythm on the same 24-h Holter ECG recording. In 14 patients, antiarrhythmic drugs (AAD) were administered. QT/RR relations were analyzed from ECG waves obtained by the summation of consecutive QRS-T complexes during each 15-s period (QT/RR-average) and a single beat QRS-T (QT/RRsingle). During sinus rhythm, the slope of QT/RR-average did not differ from that of QT/RR-single in patients with and without AAD. On the other hand, during AF, the slope of QT/RR-average was significantly greater than that of $Q T / R R$-single (without AAD: $0.12 \pm 0.06$ vs. $0.06 \pm 0.03, P<0.001$; with $A A D: 0.15 \pm 0.05$ vs. $0.08 \pm 0.04, P<0.001$ ). During AF, the QT interval at an RR interval of 1.2-s (QT-1.2) determined from QT/RR-average was significantly greater than QT-1.2 from QT/RR-single in patients with and without AAD. QT-1.2 in QT/RR-single during AF was significantly smaller than that during sinus rhythm but QT-1.2 in QT/RR-average during AF was not.
\end{abstract}

Conclusions: The QT interval after sinus restoration could be estimated better using QT/RR-average than using QT/RR-single during AF. (Circ J 2011; 75: 274-279)

Key Words: Antiarrhythmic drug; Atrial fibrillation; Holter ECG; QT/RR relation

$\mathbf{T}$ he relationship between QT and RR intervals during sinus rhythm has been studied intensively. ${ }^{1-3}$ Analyses of automatic QT measurement using 24-h Holter electrocardiogram (ECG) recordings have shown that QT measurement is reliable and reproducible during sinus rhythm and adaptation of ventricular repolarization to heart rate could be assessed by the slope and intercept of the linear regression line of QT/RR relation. ${ }^{4-6}$ During sinus rhythm QT/RR relation was assessed by 2 different methods: (1) based on an averaged beat ECG and (2) based on a single beat ECG using 24-h Holter ECG.

During atrial fibrillation (AF), however, only a few reports have described different characteristics of QT/RR relation compared with sinus rhythm. ${ }^{7,8}$ Patients with AF are often treated with QT-prolonging antiarrhythmic drugs (AAD) and in some patients the QT-prolonging effects of AAD become more remarkable after sinus restoration. ${ }^{9}$ A recent study confirmed that patients with AAD therapy are more likely to develop torsades de pointes when the underlying rhythm is a sinus rhythm compared with AF. ${ }^{10}$ Hence, reliable methods for assessing QT/RR relation during AF are of clinical importance especially in patients with AAD therapy. The aim of the present study was to compare QT/RR relation based on an averaged beat ECG with a single beat ECG during AF using Holter ECG and to find out which method was better for estimation of QT interval after sinus restoration.

\section{Methods}

This study consisted of 33 patients with paroxysmal AF (Table 1). Patients were required to have episodes of both

\begin{tabular}{|lc|}
\hline Table 1. Clinical Characteristics & \\
Patients/women & $33 / 9$ \\
Age (years) & $67.2 \pm 13.3$ \\
Ischemic heart disease & 5 \\
Hypertension & 9 \\
Hypertrophic cardiomyopathy & 2 \\
$\beta$-blocker & 5 \\
Calcium antagonist & 5 \\
Antiarrhythmic drug & 14 \\
\hline
\end{tabular}

Received July 6, 2010; revised manuscript received September 21, 2010; accepted October 18, 2010; released online December 17, 2010 Time for primary review: 21 days

Division of Cardiology, Shizuoka Red Cross Hospital, Shizuoka, Japan

Mailing address: Akira Fujiki, MD, PhD, Division of Cardiology, Shizuoka Red Cross Hospital, 8-2 Otemachi, Aoi-ku, Shizuoka 4200853, Japan. E-mail: afujiki@szrc.org

ISSN-1346-9843 doi:10.1253/circj.CJ-10-0654

All rights are reserved to the Japanese Circulation Society. For permissions, please e-mail: cj@j-circ.or.jp 


\section{A 15 -s averaged beat analysis}

QT

(s)

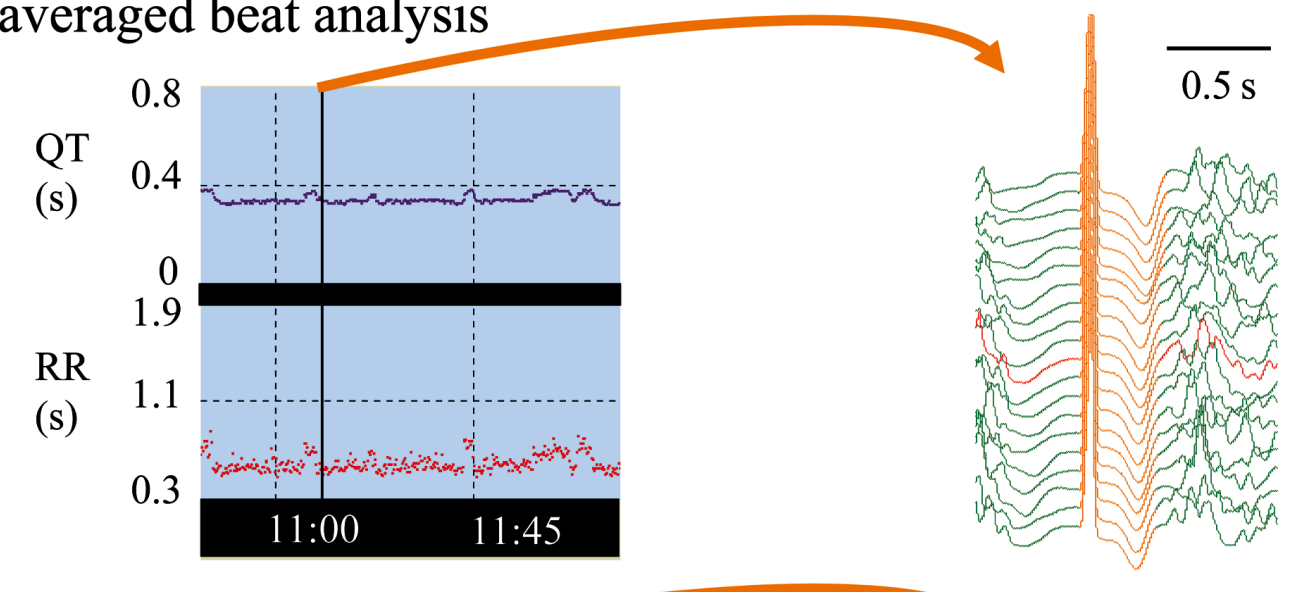

\section{A single beat analysis}

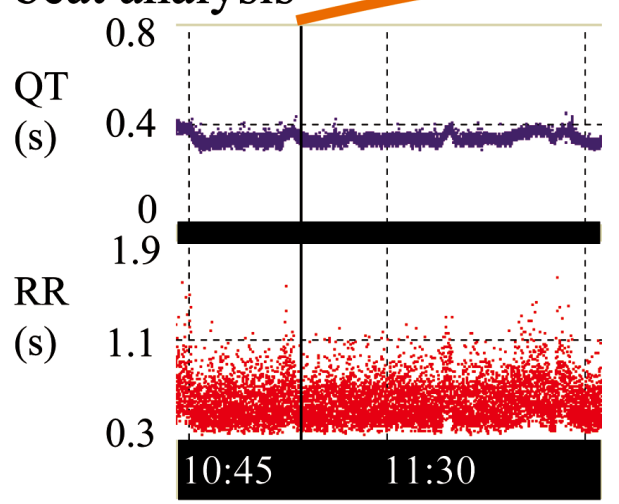

Figure 1. QT and RR intervals based on a 15-s averaged beat analysis (Upper) and a single beat analysis (Lower) during AF. Right panels show representative 20 consecutive beats of QRS-T complex during AF based on a 15-s averaged beat analysis (Upper) and a single beat analysis (Lower). Orange line indicates an automatically determined QT interval. AF, atrial fibrillation.

$\mathrm{AF}$ and sinus rhythm after AF termination on the same 24-h Holter ECG, where the duration of each AF episode was longer than at least $60 \mathrm{~min}$. The subjects consisted of 19 patients without AAD and 14 patients with QT-prolonging AADs (cibenzoline 3, sotalol 3, bepridil 4 and amiodarone 4).

Holter ECGs were recorded using NASA (the bipolar lead between the superior and inferior edge of the sternum, used by the National Aeronautics and Space Administration) and CM5 (the modified chest lead V5) leads for 24h, and CM5 lead was used for automatic QT measurements. A digital ECG recording device (FM-180, Fukuda Denshi, Tokyo, Japan) with a sampling rate of $128 / \mathrm{s}$ was used with an automatic measurement system (SCM-6600, Fukuda Denshi) and QT analyzing software (HPS-QTM, Fukuda Denshi). The analyzing system determined the top and the end of the $\mathrm{T}$ wave automatically, according to the following algorithm. The top of the $\mathrm{T}$ wave was determined as the point where the first derivative $(\mathrm{dv} / \mathrm{dt})$ of the $\mathrm{T}$ wave polarity changed. The end point of the $T$ wave was determined as the point where the first derivative of the $T$ wave became undetectable after the top of the T wave. In each case, the detection level of the first derivative of the $\mathrm{T}$ wave was set as the average level of the ST segment to overcome the background noise.

The recordings with periods of $\mathrm{AF}$ and sinus rhythm after
AF conversion were analyzed separately and the slope and intercept of QT/RR relation were obtained using 2 different methods. One method plotted the QT interval against the RR interval from averaged ECG waves obtained by the summation of consecutive QRS-T complexes during each 15-s period over several hours (QT/RR-average). QT and RR intervals based on a 15-s averaged beat analysis are shown in Figure 1 (Upper panels). Averaging the time series of 15-s segments during AF decreased the variability of QT and RR intervals. The other method plotted the QT interval of each single beat against the previous RR interval and correlated without any preprocessing for several hours (QT/RR-single). QT and RR intervals based on a single beat analysis are shown in Figure 1 (Lower panels). The variability of QT intervals was remarkable due to changes in RR intervals during AF.

\section{Statistical Analysis}

Results are presented as mean \pm standard deviation (SD). The dependence of the QT interval on the RR interval was analyzed by linear regression in each patient $(\mathrm{QT}=\mathrm{A}[\mathrm{RR}]+\mathrm{B}$; where $A$ is the slope and $B$ is the intercept). Unpaired and paired data were analyzed by Student's t-test. Comparisons of multiple groups were obtained by ANOVA with Fisher's protected least significant difference. Yates $2 \times 2$ chi-square 

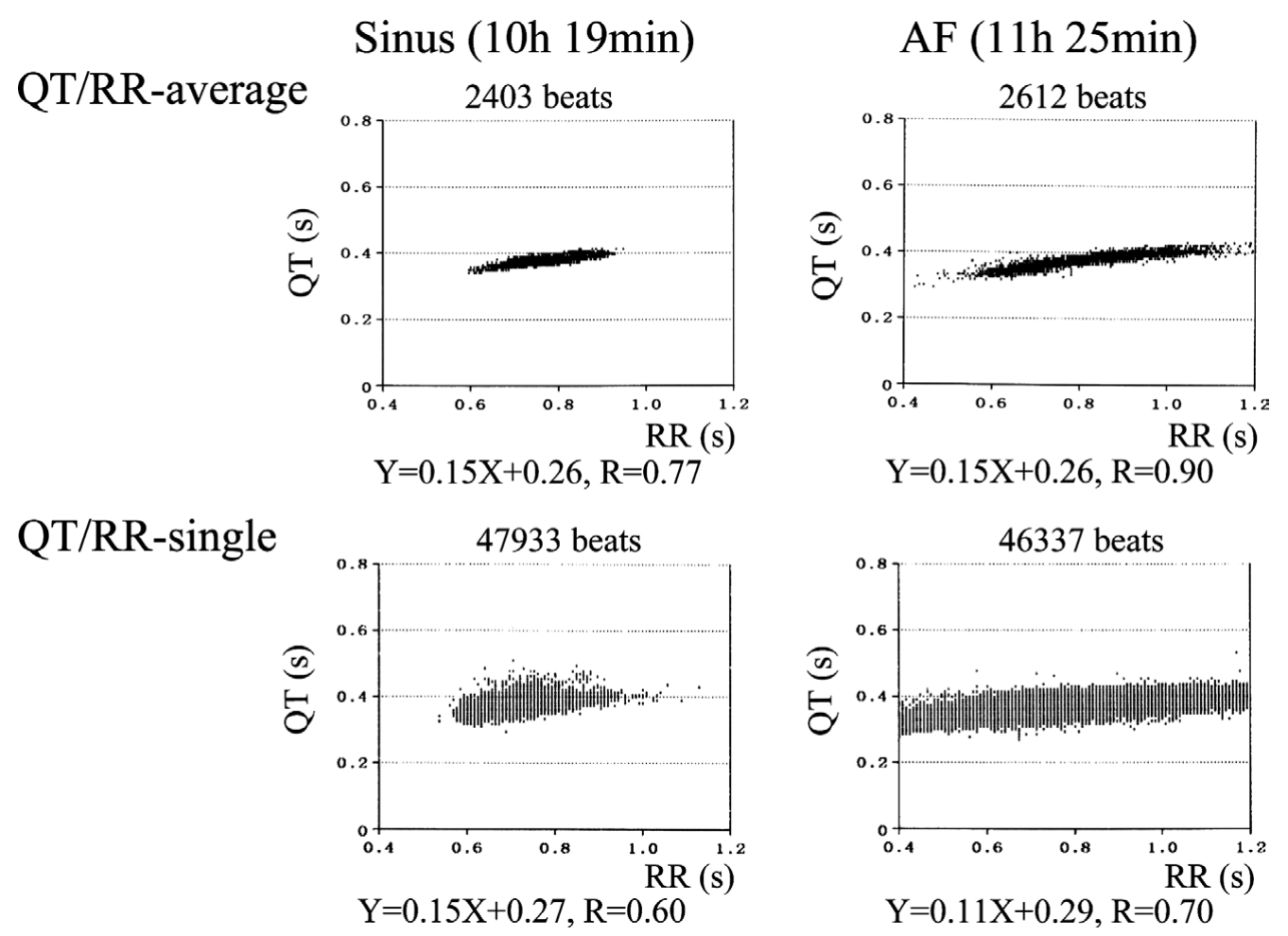

Figure 2. QT/RR relations during sinus rhythm and atrial fibrillation (AF) in a patient without antiarrhythmic drug therapy. (Upper) QT/RR-average; (Lower) QT/RR-single.
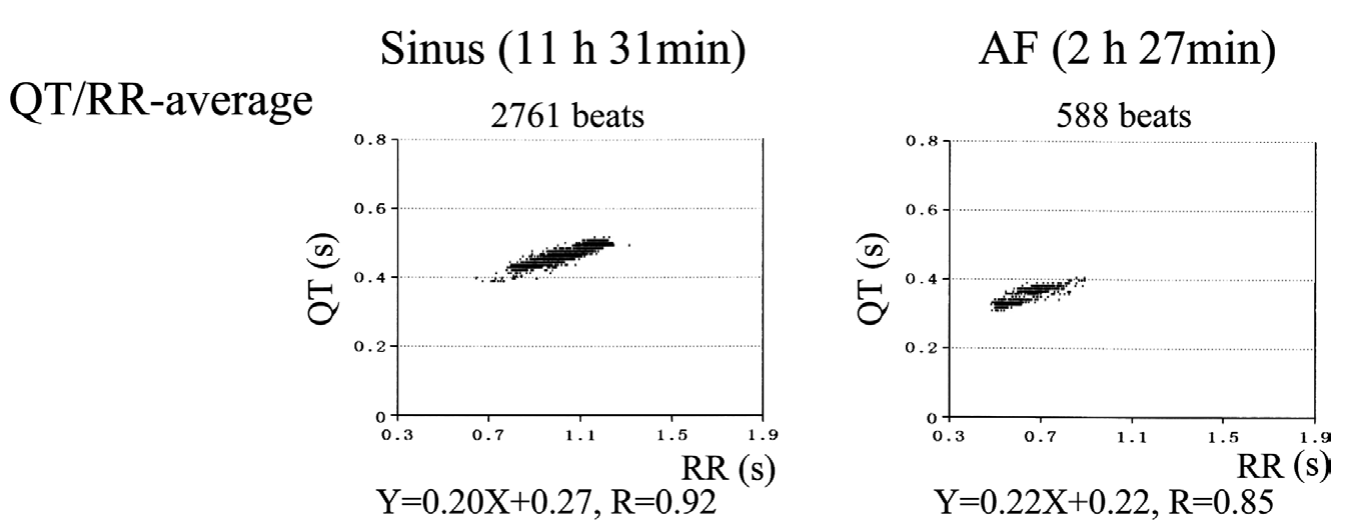

QT/RR-single
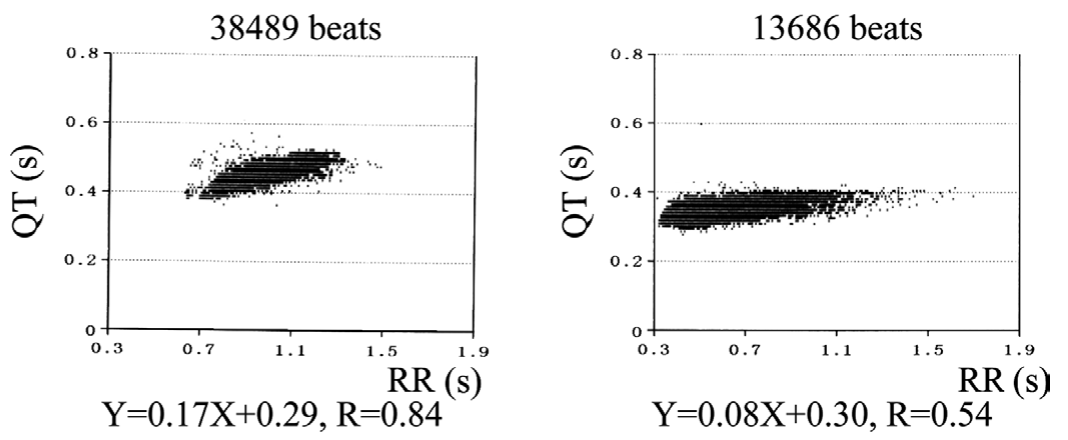

Figure 3. QT/RR relations during sinus rhythm and atrial fibrillation (AF) in a patient with antiarrhythmic drug therapy (amiodarone $200 \mathrm{mg} /$ day). (Upper) QT/RR-average; (Lower) QT/RR-single. 


\begin{tabular}{|c|c|c|c|c|}
\hline & \multicolumn{2}{|c|}{ No antiarrhythmic drugs $(n=19)$} & \multicolumn{2}{|c|}{ Antiarrhythmic drugs $(n=14)$} \\
\hline & Slope & Intercept & Slope & Intercept \\
\hline \multicolumn{5}{|l|}{ Sinus rhythm } \\
\hline QT/RR-average & $0.13 \pm 0.04^{*}$ & $0.28 \pm 0.04$ & $0.20 \pm 0.05^{\star, \#}$ & $0.27 \pm 0.09$ \\
\hline QT/RR-single & $0.12 \pm 0.05^{\star}$ & $0.29 \pm 0.04$ & $0.18 \pm 0.05^{\star}, \#$ & $0.29 \pm 0.07$ \\
\hline \multicolumn{5}{|l|}{ AF } \\
\hline QT/RR-average & $0.12 \pm 0.06^{*}$ & $0.27 \pm 0.04$ & $0.15 \pm 0.05^{\star}$ & $0.28 \pm 0.06$ \\
\hline QT/RR-single & $0.06 \pm 0.03$ & $0.31 \pm 0.02$ & $0.08 \pm 0.04$ & $0.32 \pm 0.04$ \\
\hline
\end{tabular}

Values are mean \pm SD.

${ }^{*} \mathrm{P}<0.001$ vs. $\mathrm{QT} / \mathrm{RR}$-single during $\mathrm{AF},{ }^{\#} \mathrm{P}<0.001$ vs. no antiarrhythmic drug group.

$\mathrm{AF}$, atrial fibrillation.
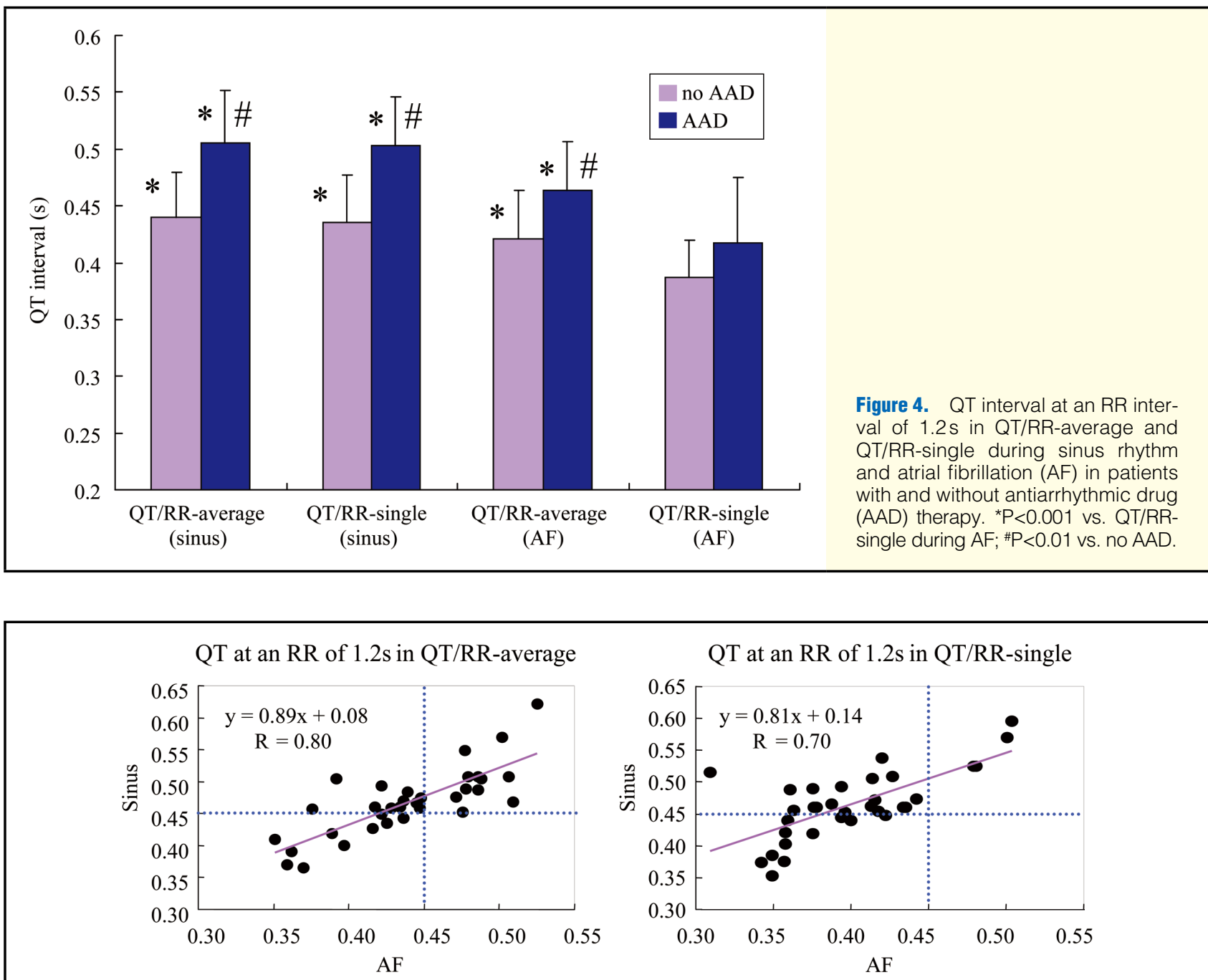

Figure 5. Relationship between QT interval at an RR interval of 1.2s (QT-1.2) in QT/RR-average (Left) or QT/RR-single (Right) during atrial fibrillation (AF) and during post cardioversion sinus rhythm. Prolonged QT-1.2 during post cardioversion sinus rhythm $(\geq 0.45 \mathrm{~s})$ could be better estimated using QT/RR-average $(\chi=8.19, P<0.01)$ than using QT/RR-single.

test was used to compare the categorical variables between groups. Statistical significance was set at $\mathrm{P}<0.05$.

\section{Results}

The clinical characteristics of patients are summarized in
Table 1. Representative QT/RR-average and QT/RR-single during sinus rhythm and during AF are shown in Figure 2 (without AAD) and Figure 3 (with amiodarone). In 19 patients without AAD, during sinus rhythm, the slope of QT/RRaverage did not differ from that of QT/RR-single (Table 2). During AF, the slope of QT/RR-average $(0.12 \pm 0.06)$ was 
significantly greater than that of QT/RR-single $(0.06 \pm 0.03$, $\mathrm{P}<0.001)$. The slope of QT/RR-single during AF $(0.06 \pm 0.03)$ was significantly smaller than that during sinus rhythm $(0.12 \pm$ $0.05, \mathrm{P}<0.001)$.

In 14 patients with $\mathrm{AAD}$ therapy, the slopes of regression line in QT/RR-average and QT/RR-single during sinus rhythm were steeper compared with those in 19 patients without AAD therapy (Table 2). During AF, the slope of QT/RR-average $(0.15 \pm 0.05)$ was significantly greater than that of QT/RR-single $(0.08 \pm 0.04, \mathrm{P}<0.001)$. The slope of $\mathrm{QT} / \mathrm{RR}$-single during AF $(0.08 \pm 0.04)$ was significantly smaller than that during sinus rhythm $(0.18 \pm 0.05, \mathrm{P}<0.001)$. The slope of QT/RR-average during AF had a tendency to be smaller than that during sinus rhythm but the difference was statistically insignificant.

During AF or sinus rhythm, the QT interval at an RR interval of 1.2-s (QT-1.2) in QT/RR-average or QT/RR-single was significantly greater in patients with AAD compared with those without AAD except QT-1.2 in QT/RR-single during AF (Figure 4). In patients with and without AAD, QT-1.2 during AF was significantly smaller in QT/RR-single than in QT/RR-average. QT-1.2 in QT/RR-single was significantly smaller during AF than during sinus rhythm.

In patients with or without AAD, QT-1.2 during AF determined from QT/RR-average (Figure 5 Left) and from QT/RR-single (Figure 5 Right) correlated positively with QT-1.2 during sinus rhythm. In 23 patients, QT-1.2 after sinus restoration showed $\geq 0.45 \mathrm{~s}$ and, in 12 of these patients, QT-1.2 determined from QT/RR-average during AF showed $\geq 0.45 \mathrm{~s}$ (Figure 5 Left). On the other hand, QT-1.2 determined from QT/RR-single during AF showed $\geq 0.45 \mathrm{~s}$ in only 4 patients (Figure 5 Right). Prolonged QT-1.2 ( $\geq 0.45 \mathrm{~s})$ during post cardioversion sinus rhythm could be identified better using QT/RR-average $(\chi=8.19, \mathrm{P}<0.01)$ than using QT/RR-single.

\section{Discussion}

During AF, the slope of QT/RR-average was significantly greater than that of QT/RR-single in patients with and without AAD therapy. The slope of QT/RR-single was significantly smaller during AF than during sinus rhythm. During AF, QT1.2 in QT/RR-average was significantly greater than QT-1.2 in QT/RR-single in patients with and without AAD. QT-1.2 in QT/RR-single was significantly greater during sinus rhythm than during AF but QT-1.2 in QT/RR-average during sinus rhythm was similar to that during AF. The QT interval after sinus restoration could be estimated better using QT/RRaverage than QT/RR-single during AF.

\section{QT/RR During AF}

The QT interval changes along with the heart rate and it is corrected using several formulae, such as Bazett and Fridericia. ${ }^{11}$ However, standard "rate-correction" formulae underestimate the change in QT at slower heart rates during sinus rhythm. ${ }^{12,13}$ Furthermore, there are no established methods for evaluating QT intervals during AF. A new approach to analyze QT intervals during AF was proposed by Darbar et al. ${ }^{14}$ Sorting raw QT data into "bins", which are determined by the preceding RR intervals permits assessment of QT/RR relationships over a broad RR range during AF. The authors revealed that cardioversion of AF acutely prolonged the QT interval and increased the steepness of the QT/RR slope.

During AF, the second and further preceding RR intervals before the preceding RR interval may play an important role for the regulation of the QT interval. Larroude et al demonstrated that QT dynamics could be reliably measured in AF and were comparable with sinus rhythm when several preceding RR intervals were included in the analysis. ${ }^{15}$ Adaptation of the QT interval to changes in the preceding RR interval consists of 2 components: the fast component (non-steady state adaptation) and the slow component (steady state adaptation). ${ }^{16}$ In the present study, we adopted the single beat (QT/RR-single) and the 15-s averaged ECG (QT/RR-average) during AF and found that the slope of QT/RR-average was greater than that of QT/RR-single during AF and was closer to that of QT/RR during sinus rhythm. Hysteresis of paced QT interval in response to abrupt changes in pacing rate was studied by Lau et al. ${ }^{17}$ The time constant of QT adaptation was $60.4 \mathrm{~s}$ when the rate was decreasing, which may be related to the steeper slope of QT/RR based on the 15-s averaged ECG wave during AF compared with QT/RR based on a single beat. Our findings suggest that QT/RR during sinus rhythm could be estimated better using QT/RR-average than using QT/RR-single during AF.

\section{QT/RR and AAD}

Drugs that prolong the action potential duration of the atrial myocardium can suppress AF, but they have the possibility to cause excessive QT prolongation leading to torsades de pointes. ${ }^{18,19}$ Although the risk of proarrhythmia is higher after sinus restoration compared with during $\mathrm{AF},{ }^{10}$ it is sometimes difficult to estimate the degree of QT prolongation after sinus restoration. During sinus bradycardia, an increase in IKr may prevent the excessive QT prolongation after a long RR interval and also may prevent a steeper slope of QT/RR relation. AADs with an IKr blocking effect could cancel these protective effects and increase the slope of QT/RR.

In patients with and without $\mathrm{AAD}$, the QT interval after sinus conversion could be better estimated by analyzing QT/RR-average rather than QT/RR-single during AF. In patients with QT-prolonging AAD therapy for AF, not only QT morphology including notched T waves, ${ }^{20}$ but a steeper slope of QT/RR-average during AF may indicate risks of the exaggerated prolongation of the QT interval after sinus restoration.

\section{Study Limitations}

This study was performed in a limited number of patients who had no episode of torsades de pointes. The precise effects of $\mathrm{AAD}$ on $\mathrm{QT} / \mathrm{RR}$ relation during $\mathrm{AF}$ require evaluation in future studies with a much larger number of patients. The different autonomic nerve activity between sinus rhythm and AF may affect QT-RR relation differently. The linear regression line of QT/RR was obtained from relations between QT and RR during AF and sinus rhythm but the actual sampling range of the heart rate (RR intervals) was limited in each subject. In most patients, we could estimate QT/RR relation using the linear regression line within the physiological range of RR intervals.

\section{Conclusions}

The slope of QT/RR-average during AF was greater than that of QT/RR-single during AF and was not different from that of QT/RR after sinus rhythm restoration. The QT interval during sinus rhythm could be estimated better using QT/RRaverage compared with QT/RR-single during AF.

\section{References}

1. Malik M, Färbom P, Batchvarov V, Hnatkova K, Camm AJ. Relation between QT and RR intervals is highly individual among 
healthy subjects: Implications for heart rate correction of the QT interval. Heart 2002; 87: 220-228.

2. Toivonen L. More light on QT interval measurement. Heart 2002; 87: $193-194$.

3. Al-Khatib SM, LaPointe NM, Kramer JM, Califf RM. What clinicians should know about the QT interval. JAMA 2003; 289: 2120 2127.

4. Merri M, Moss AJ, Benhorin J, Locati EH, Alberti M, Badilini F. Relation between ventricular repolarization duration and cardiac cycle length during 24-Holter recordings: Findings in normal patients and with long QT syndrome. Circulation 1992; 85: 18161821.

5. Emori T, Ohe T, Aihara N, Shimizu W, Kamakura S, Shimomura $\mathrm{K}$. Dynamic relationship between the QaT interval and heart rate in patients with long QT syndrome during 24-hour Holter ECG monitoring. Pacing Clin Electrophysiol 1995; 18: 1909-1918.

6. Fujiki A, Sugao M, Nishida K, Sakabe M, Tsuneda T, Mizumaki K, et al. Repolarization abnormality in idiopathic ventricular fibrillation: Assessment using 24-hour QT-RR and QaT-RR relationships. J Cardiovasc Electrophysiol 2004; 15: 59-63.

7. Pai GR, Rawles JM. The QT interval in atrial fibrillation. Br Heart J 1989; 61: 510-513.

8. Ehlert FA, Goldberger JJ, Rosenthal JE, Kadish AH. Relation between QT and RR intervals during exercise testing in atrial fibrillation. Am J Cardiol 1992; 70: 332-338.

9. Choy AM, Darbar D, Dell'Orto S, Roden DM. Exaggerated QT prolongation after cardioversion of atrial fibrillation. J Am Coll Cardiol 1999; 34: 396-401.

10. Darbar D, Kimbrough J, Jawaid A, McCray R, Ritchie MD, Roden DM. Persistent atrial fibrillation is associated with reduced risk of torsades de pointes in patients with drug-induced long QT syndrome. $J$ Am Coll Cardiol 2008; 51: 836-842.

11. Indik JH, Pearson EC, Fried K, Woosley RL. Bazett and Fridericia QT correction formulas interfere with measurement of drug-induced changes in QT interval. Heart Rhythm 2006; 3: 1003-1007.
12. Sugao M, Fujiki A, Sakabe M, Nishida K, Tsuneda T, Iwamoto J, et al. New quantitative methods for evaluation of dynamic changes in QT interval on 24 hour Holter ECG recordings: QT interval in idiopathic ventricular fibrillation and long QT syndrome. Heart 2006; 92: 201-207.

13. Woosley RL, Romero K. Time to replace Bazett's QT-correction. Pacing Clin Electrophysiol 2009; 32: 1379-1380.

14. Darbar D, Hardin B, Harris P, Roden DM. A rate-independent method of assessing QT-RR slope following conversion of atrial fibrillation. J Cardiovasc Electrophysiol 2007; 18: 636-641.

15. Larroude CE, Jensen BT, Agner E, Toft E, Torp-Pedersen C, Wachtell K, et al. Beat-to-beat QT dynamics in paroxysmal atrial fibrillation. Heart Rhythm 2006; 3: 660-664.

16. Franz MR, Swerdlow CD, Liem LB, Schaefer J. Cycle length dependence of human action potential duration in vivo: Effects of single extrastimuli, sudden sustained rate acceleration and deceleration, and different steady-state frequencies. J Clin Invest 1988; 82: $972-979$.

17. Lau CP, Freedman AR, Fleming S, Malik M, Camm AJ, Ward DE. Hysteresis of the ventricular paced QT interval in response to abrupt changes in pacing rate. Cardiovasc Res 1988; 22: 67-72.

18. Yamashita T, Ogawa S, Sato T, Aizawa Y, Atarashi H, Fujiki A, et al. Dose-response effects of bepridil in patients with persistent atrial fibrillation monitored with transtelephonic electrocardiograms: A multicenter, randomized, placebo-controlled, double-blind study (J-BAF Study). Circ J 2009; 73: 1020-1027.

19. Kurokawa S, Niwano S, Kiryu M, Murakami M, Ishikawa S, Yumoto Y, et al. Importance of morphological changes in T-U waves during bepridil therapy as a predictor of ventricular arrhythmic event. Circ J 2010; 74: 876-884.

20. Topilski I, Rogowski O, Rosso R, Justo D, Copperman Y, Glikson $\mathrm{M}$, et al. The morphology of the QT interval predicts torsade de pointes during acquired bradyarrhythmias. J Am Coll Cardiol 2007; 49: $320-328$. 